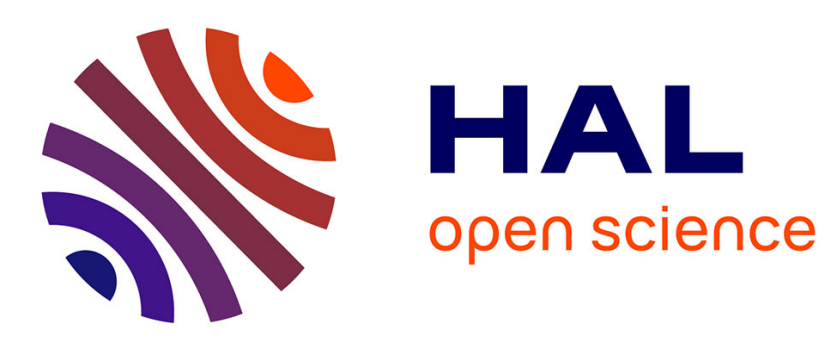

\title{
Cooperation between business and holonic manufacturing decision systems
}

Rémi Pannequin, André Thomas

\section{To cite this version:}

Rémi Pannequin, André Thomas. Cooperation between business and holonic manufacturing decision systems. 12th IFAC Symposium on Information Control Problems in Manufacturing INCOM'2006, May 17-19, 2006, St Etienne, France. pp.423-428. hal-00081846

\section{HAL Id: hal-00081846 https://hal.science/hal-00081846}

Submitted on 26 Jun 2006

HAL is a multi-disciplinary open access archive for the deposit and dissemination of scientific research documents, whether they are published or not. The documents may come from teaching and research institutions in France or abroad, or from public or private research centers.
L'archive ouverte pluridisciplinaire HAL, est destinée au dépôt et à la diffusion de documents scientifiques de niveau recherche, publiés ou non, émanant des établissements d'enseignement et de recherche français ou étrangers, des laboratoires publics ou privés. 


\title{
COOPERATION BETWEEN BUSINESS AND HOLONIC MANUFACTURING DECISION SYSTEMS
}

\author{
Rémi Pannequin, André Thomas
}

\author{
Research Centre for Automatic Control CNRS UMR 7029, \\ Campus scientifique, 54506 Vandouvre Cedex, France \\ \{remi.pannequin, andre.thomas\}@cran.uhp-nancy.fr
}

\begin{abstract}
New developments in production control can be grouped around two modes: distributed and centralised control. Interaction between these control modes can be seen as a B2M issue. We propose in this paper an alternative B2M architecture, using holonic products, in order to enable cooperation between centralised business and distributed manufacturing decision systems. We will first give the requirements of such a system, then outline the structure of the holonic product and finally study the possible interaction protocols between the products and the decision system.
\end{abstract}

Keywords: holonic control, product-driven systems, B2M architecture

\section{INTRODUCTION}

In the area of enterprise information system design, the business to manufacturing (B2M) interface has generated a lot of research. Among the many aspects of $\mathrm{B} 2 \mathrm{M}$, we focus on production control concerns. Indeed, enterprises face new challenges, and evolutions of control systems are required. On the one hand, global performance is needed, not only considering the enterprise, but also the supply chain. On the other hand agility is required, in the context of shorter product life-cycle and increasing product variety.

One of the trends in production control consists in improving existing manufacturing production planning and control (PPC) systems. For instance, advanced planning systems (APS) are based on the mature control architecture MRP2 (Manufacturing Resources Planning) (Vollman et al., 1997). These tools use advanced operational research algorithms to provide forecasting, planning and scheduling functions. Using the integration paradigm, they centralise information about the whole supply chain, for every planning level. Therefore they aim at a global optimisation of production.
Another trend consists in the use of distributed, intelligent control systems, like holonic manufacturing systems (HMS). The holonic paradigm (Valckenaers, 2001) consists in distributing technical intelligence, or even production control decisions among the actual elements of the manufacturing system. For instance, the PROSA reference architecture deals with resources, products and order agents (van Brussel et al., 1998). These intelligent systems use control paradigms from distributed artificial intelligence (DAI) rather than operational research algorithms. Indeed, the global control behaviour emerges from local decisions and from interactions (synchronisation, data exchange, negotiation) between these holonic entities.

Besides, advances in automatic identification, in particular radio-frequency identification (RFID), enable to associate some easily-accessible data to a product. Using RFID tags, the product might become an information-storing and processing item. Then, this smart product can be connected to the enterprise information system, or even to the global network, constituting an "Internet of things" (McFarlane et al., 2003).

This lead us to envisage product-driven systems (Morel et al., 2003). In such systems, products are active 
objects, which control their own mobility in the production system. The expected benefits of this new approach concern flexibility and robustness of control, support of high product variety in production flows (mass-customisation), or traceability along the supply chain. We can therefore distinguish two main control modes, one centralised and the other distributed. Both have advantages and drawbacks, and they may coexist in the same organisation. So, our problem is the interaction between centralised and distributed control modes. From the point of view of control, the key issue is to combine optimality of centralised control with flexibility of distributed control. From the point of view of information system engineering, the main concern is to define an architecture in which both types of control systems could cooperate and evolve.

In this paper we propose to use smart-products to enable cooperation between centralised and distributed control systems.

First, both control modes will have to be more precisely characterised. Then, we will show how interactions between these control modes can be seen as a $\mathrm{B} 2 \mathrm{M}$ issue, and propose a product-driven B2M architecture. Finally, we will present a model of the system.

\section{CHARACTERISATION OF CENTRALISED AND DISTRIBUTED CONTROL MODES}

\subsection{Hierarchical decomposition of decision systems}

The enterprise decision system may be modelled using a hierarchical decomposition. This refers to the well known CIM (computer integrated manufacturing) model (ISO, 2002). The control functions are classified according to information aggregation level, to weaker real-time constraints, and also to a broader scope.

This hierarchical decomposition came first from the study and modelling of existing (hierarchical) decision systems. But it also shows a more general structure of enterprises, related to the cognitive limit of each element (Simon, 1996). Actually, similar decompositions can be found in other domains, for instance to achieve control of autonomous vehicles (Albus and Barbera, 2005). Indeed, a broader scope for decisionmaking implies higher aggregation of information, and weaker real-time constraints.

Therefore, we can use the CIM model as a kind of map, where decision centres (DC) possibly not hierarchically organised can be positioned. This will help us in the study of decision systems.

An important characteristic is the existence of central elements. A central element is a decision centre that manages "lower levels" DC. A decision system without central element is "heterarchical" (Bongaerts et al., 2000). On the contrary, the use of central elements is the base of hierarchy. Nevertheless, this structural point of view is not sufficient to characterise the organisation of a decision system, "ranging from hierarchical to non-hierarchical control" (Brennan and Norrie, 2003).

The other aspects that must be taken into account are the nature of the relationship between DC, and their dynamic behaviour.

\subsection{Nature of relationships}

Information received by a DC reduces its freedom to make a choice and therefore impacts on the distribution of decisions.

First, the relation depends on the role associated to the transmitted information. For instance, in the case of a master-slave relationship, this role is imperative. A piece of information received (e.g. a production schedule) must be executed without being examined. The role can also be informative if the receiver is given some autonomy with respect to the transmitted information. These two kinds of relations, respectively called "hierarchical" and "unconstrained-hierarchical" have been implemented and compared (Brennan and Norrie, 2003).

Then it also depends of the nature of decisions taken by central DC. If they are highly detailed, then freedom of lower DC is reduced. If it is aggregated or in another way imprecise, like in a fuzzy plan (Grabot and Geneste, 1998), then the domain of choice is broader. For instance, executing fuzzy plans empowers operators, who have to take scheduling decisions, making the decision system more distributed.

So, depending on the kind of relationships that a central element have with lower level DC, the system can be either centralised (every decision is taken by the centre, lower levels only "repeat" what have been beforehand decided), or distributed, if central elements have only an informative role.

\subsection{Dynamic behaviour}

A first approach to the dynamics of decision processes is based on a periodic execution of decision tasks. This is indeed true for high level planning decisions (MRP2). For example, the master production schedule is usually elaborated once a week, for a time horizon equal to the cumulative lead time. So, like in the GRAI enterprise modelling methodology (Doumeingts, 1984), DC dynamics can be described by a periodicity, and a time horizon.

Nevertheless, this characterisation only fit a one-shot problem solving behaviour. In this way to solve problems, decisions are produced according to mathematical representations of the present state of the production system, and to predictions (or assumptions) of 
its evolutions. The decisions come from operationalresearch algorithms, and are optimal with respect to this model. As this optimisation procedure require much computer time, it is only executed periodically, or when events occur.

On the contrary, intelligent systems solve on-going concerns (Valckenaers et al., 2003). They try to maintain the production system in its living condition, by continuously adjusting its parameters, reacting to events coming from workshop equipments, from other enterprise applications. Solving on-going concerns can also be proactive, if effects of the decisions in the future are taken into account. An example of such a decision system is the ants-based production control system developed at KU Leuven (Hadeli et al., 2004).

To summarise, we distinguish two control modes (table 1). Centralised control, global and predictive, is widely used for business applications. Distributed control, local and reactive, can be applicated to manufacturing control.

$\underline{\text { Table 1. Centralised and distributed control }}$ characteristics

\begin{tabular}{lcc} 
control mode & centralised & distributed \\
\hline scope & global & local \\
behaviour & predictive & reactive \\
problem solving & one-shot & on-going \\
paradigm & & \\
\hline
\end{tabular}

\section{CENTRALISED VS. DISTRIBUTED CONTROL AS A B2M ISSUE}

\subsection{Classical B2M model}

In the classical architecture, business and manufacturing domains are defined according to a hierarchical decomposition of the control system. Business decisions are associated to the higher levels of the pyramid, whereas lower levels are concerned by manufacturing decisions (ISO, 2002).

Both domains are integrated, and maintain each an informational image of the physical flow of products (figure 1). Therefore, there are three different flows:

- the physical products,

- information representing products from the manufacturing point of view,

- information representing products from the business point of view.

Synchronising information about the product is the task of the B2M layer. Nevertheless, in this architecture, it is hard for a (possibly distributed) manufacturing decision to take place. On the one hand, increasing computing capabilities tend to produce more and more precise decisions, using for instance APS or scheduling applications. On the other hand, if manufacturing decision centres are given some autonomy, local decisions may cause inconsistencies.

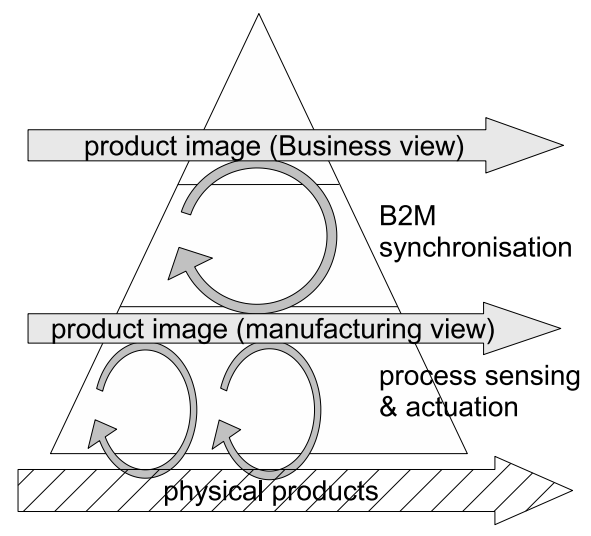

Fig. 1. Traditional B2M architecture

Besides, a very precise and timely accurate image of the workshop must be available, in order to fulfil new requirements on business, like on-demand production, mass customisation, or the ability for a customer to track the progress of his order.

\subsection{Holonic products to solve B2M interaction}

Instead of trying to integrate business and manufacturing domains, we accept their existence as autonomous entities, and try to make them cooperate with "intelligence".

Business and manufacturing activities differ by their view of the enterprise goal. On the one hand, business concerns are related to the satisfaction of a market, taking into account financial constraints (e.g. cashflow). On the other hand manufacturing concerns are related to the production of physical goods, taking into account technical constraints.

The product is the common object shared by both business and manufacturing decision systems. Therefore, we try to implement B2M cooperation by merging business and manufacturing information about the product into the product itself. From the assembly of these information and physical flows emerge a flow of holonic products (Gouyon et al., 2004).

So, in this architecture, two autonomous decision systems, dealing with different production control concerns, will cooperate through a flow of holonic products (figure 2).

\subsection{Production control functions}

The business decision system will use centralised control to plan ahead, in order to fulfil customer's demands. This consists in making:

- long-term strategic decisions,

- sales and operations plan (usually monthly production plan for family of products, in each site, or across the supply chain), 


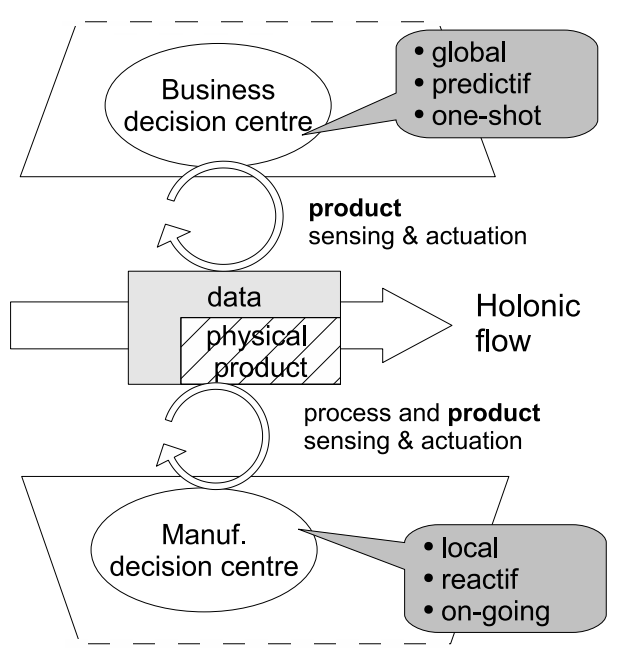

Fig. 2. Alternative product-driven B2M architecture (Morel et al., 2005)

- master production schedule (usually weekly production plan for products, in each site)

- material requirement planning,

- individual orders tracking/tracing.

The manufacturing decision system may use distributed control, in order to maintain production system in "good conditions". This includes throughput maximisation, fastest delivery of orders, quality assurance. Manufacturing decisions possibly includes:

- workshop or site re-scheduling,

- alternate routings of product flows (e.g. in case of machine breakdown),

- lot splitting or grouping (dealing with setup times).

So decisions that result from business-oriented production planning are embedded into the flow of products. This information may be used by an autonomous manufacturing decision centre, to make its decision making-process more accurate.

Conversely, the business decision support system may observe the actual product flow. If deviation between current and predicted behaviours is too high, a rescheduling procedure might be triggered.

In conclusion, main aspects of B2M cooperation, like transmitting production schedule from business to manufacturing, or notifying about production state (usually from manufacturing to business) might be done flexibly through the actual product flow. We will now propose an infrasctructure, to enable this B2M architecture.

\section{ARCHITECTURE FOR PRODUCT-DRIVEN $\mathrm{B} 2 \mathrm{M}$}

\subsection{Functional specification}

The purpose of the system is cooperation between business and manufacturing decisions systems, using products. This main use case can be refined in three sub-cases (figure 3):

- holonic product actuation, where data is sent to the holon, either to initialise or to update its informational attributes. These attributes may be related to the "present" of the product, to its "future" i.e. the way it must be manufactured, or to its "past" i.e. what happened to it.

- holonic product sensing, where data come from the holon to a decision system, either to track its current or past states, or to get information about the way to produce it.

- synchronisation with a physical product, where the holon current state is updated to follow evolutions of the physical product.

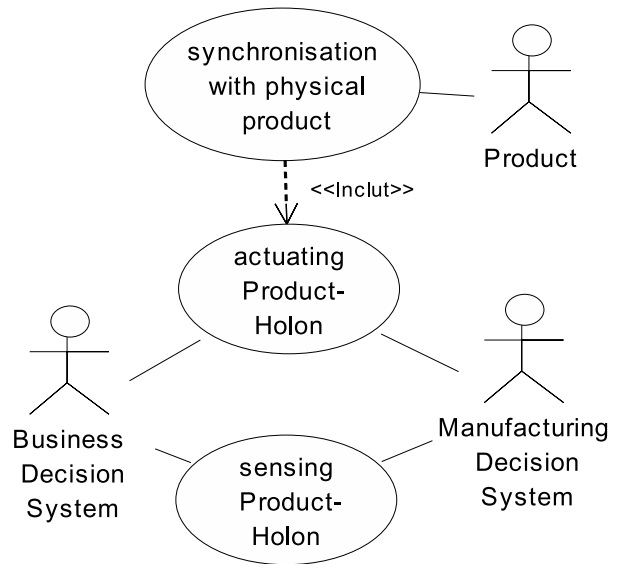

Fig. 3. UML use case diagram of product-driven B2M

\subsection{Product model}

According to the functional requirements, the holonic product must contain information about its current state, and technical information stating how to manufacture it, and what was done to it.

First, the holonic product must stay synchronised with the current state the physical product. According to systemics, the life cycle of any product can be modelled as continuous temporal, morphological or spatial transformations. We assume that these transformations can be modelled as discrete processes. For instance, a product moving by a forklift is represented only as a discrete space transformation from start to end point. This modelling comply with the discrete nature of products observation (through a finite number of sensors), and product actuation (through discrete numerically controlled processes).

Therefore, a key attribute of holonic products is a finite state machine (FSM), representing possible evolution in its life-cycle. Indeed, event coming from physical product sensing may induce change of the FSM active state, enabling synchronisation between holonic product and physical product. 
Then, holonic products must embed any other technical contents required to implement B2M cooperation. This might includes data about routing, transforming and scheduling products. To keep product model small and simple, all these contents are included into the product model using their string representation. Serialisation utilities such as XML, may be used to encode complex content, providing scalability.

Finally, since attributes may be accessed not only by their owner but also transmitted to peers, they must have unique identifiers. This identifier include holon name and attribute name, but also holon type.

Indeed, in the scope of a broader holonic manufacturing system, where resource holons may coexist with product holons, attributes pertaining to one or the other category must be distinguishable.

A class diagram, summarising the components of the product informational structure is shown figure 4.

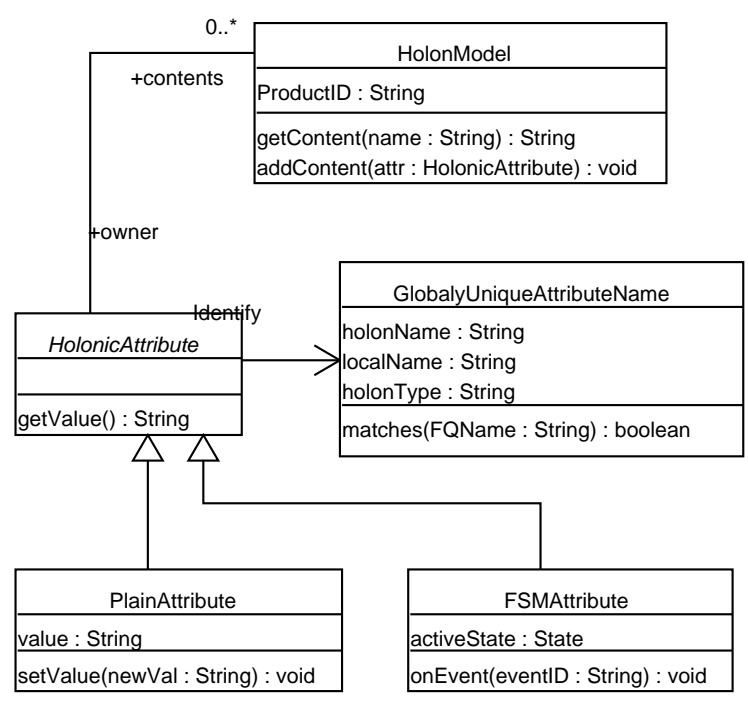

Fig. 4. Product-holon structure

\subsection{Product interaction mode}

We choose to model interactions between a holon and a decision centre, pertaining to either the manufacturing or the business decision system. Besides the exchanged content, we study the protocol used between the two peers. The foundation for intelligent physical agents (FIPA) has defined several interaction protocols.

Sending an actuation message to a product holon is not really an issue: indeed this interaction is not ambiguous, and can be done using one of the fiparequest protocols. Conversely there are several ways to define the product sensing interaction.

In the traditional resource-oriented control scheme, decision-making process is triggered by changes in resource states, and carried out by a manufacturing or business decision centre.
If product data are needed by the decision-making process they are obtained using an inspection procedure. This interaction can be implemented using the query interaction. We can notice that a decision centre don't query a particular product (e.g. by its name, or unique product code), but the product at a given location. Therefore, errors about products localisation may have high impact on this procedure.

$\underline{\text { Table 2. Protocols to observe product holons flow }}$

\begin{tabular}{lcc}
$\begin{array}{c}\text { initiator of } \\
\text { the decision }\end{array}$ & decision-maker & protocol \\
\hline resource & resource & query \\
product & resource & subscribe \\
product & product & $\begin{array}{c}\text { contract net, } \\
\text { auction }\end{array}$ \\
\hline
\end{tabular}

Another possibility is to grant products the possibility to initiate decisions. So, changes in the value of an attribute of the product holon trigger the decisionmaking process.

If products don't support the actual decision-making process, they will notify an external decision centre, using for instance the fipa-subscribe protocol. This implies that every attribute of the product holons have been beforehand declared, to enable decision centres to subscribe to them.

If the decision-making process is carried out by the product itself, it will first get information about the abilities of resources to support its needs, and finally decide about the best proposition. A way to implement this kind of negotiation is to use either the fipacontract net protocol, or one the auction protocol.

The possible interaction schemes for observing the flow of holonic products are summarised in table 2 . So, it is possible for the product to encompass the whole decision chain. But we rather see the product as a support of an external decision process, by initiating decisions, and providing decision aid information. This fits better with the technical and human reality of industry.

\subsection{Implementation using agents}

This abstract architecture is currently being implemented using the JADE (Java Agent DEvelopment framework), a FIPA-compliant framework for agent development. Product-holons have been implemented as agents. Every product-agent is given behaviours that enable him to interact with other agents, and to receive events from sensors (figure 5).

External decision-making entities have been represented in this platform using agents. Reactive and distributed decisions are taken by rule-based agents, which enforce each a particular rule. Predictive and central decisions are made by other agents, being interfaced with external scheduling tools. Finally, 


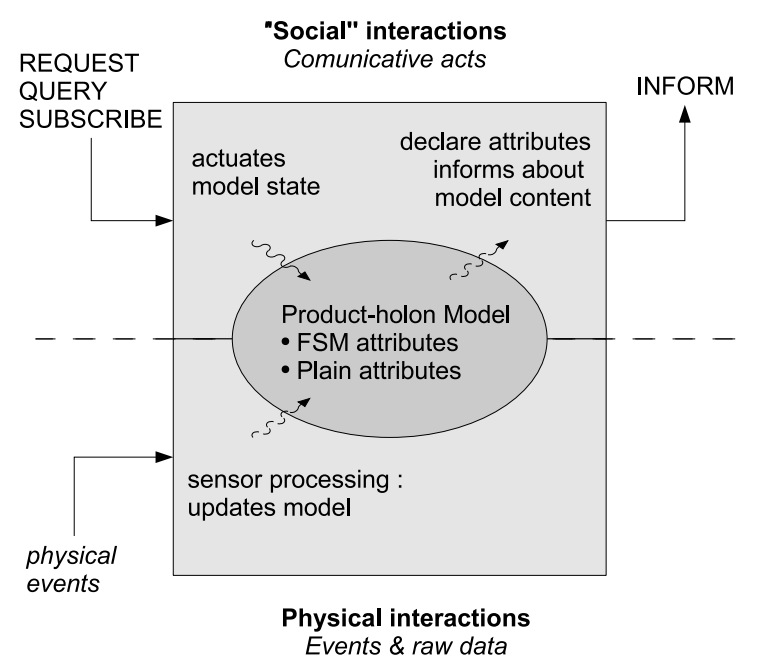

Fig. 5. Schematic view of the agent representing a holonic product

human-made decisions can be made through interfaceagents.

Moreover, the agent platform also receives physical events. To avoid technical and cost issues, an emulation of the physical process is used instead of real equipments (Pannequin and Thomas, 2006).

Preliminary experiments using this platform showed technical feasibility of the proposed architecture. Nevertheless, more work is required to obtain quantitative performance assessment.

\section{CONCLUSION}

In conclusion, we have presented a conceptual model for cooperation between two autonomous decision support systems: the business DSS, predictive and centralised, and the distributed and reactive manufacturing DSS. Instead of the traditional B2M bridge, we have proposed a cooperation scheme through holonic products. An infrastructure has been presented, that deals with storing generic informational attributes on products, and with interaction of the product with its decisional and physical environment. Implementation of this architecture using a multi-agent platform showed qualitative validation of this architecture.

As perspective, the main concern to be studied is what actual contents have to be put on the product. These contents have to be relevant with respect to business and manufacturing decision processes, to provide an efficient decision aid. Evaluating their significance would be done using our platform. These experiments may also enable a more precise validation of the concepts presented in this paper.

\section{REFERENCES}

ISO/IEC 62264 (2002). Enterprise-control system integration, part 1. model and terminology. Technical report. ISO.
Albus, J. S. and A. J. Barbera (2005). Rcs: A cognitive architecture for intelligent multi-agent systems. IFAC Annual Review in Control 29, 87-99.

Bongaerts, L., L. Monostori, D. McFarlane and B. Kádár (2000). Hierarchy in distributed shop floor control. Computers in Industry 43, 123127.

Brennan, R.W. and D.H. Norrie (2003). Metrics for evaluating distributed manufacturing control systems. Computer in Industry 51, 225-235.

Doumeingts, G. (1984). Méthode GRAI Méthode de conception et de Spécification des système de productique. Thése d'état. Université de Bordeau I. (in french).

Valckenaers, P., Ed. (2001). Special isssue on holonic manufacturing systems. Computer in Industry.

Gouyon, D., J.M. Simao, K. Alkassem and G. Morel (2004). Work in progress for product driven manufacturing automation. In: Proceedings of IFAC INCOM Symposium. Salvador de Bahia, Brazil.

Grabot, B. and L. Geneste (1998). Management of imprecision and uncertainty for production activity control. Journal of Intelligent Manufacturing 9(5), 431-446.

Hadeli, P. Valckenaers, M. Kollingbaum and H. van Brussel (2004). Multi-agent coordination and control using stigmergy. Computers in Industry 53, 75-96.

McFarlane, D., S. Sarma, K. Ashton, J.L. Chirn, C.Y. Wong (2003). Auto id systems and intelligent manufacturing control. Engineering Applications of Artificial Intelligence 16, 365-376.

Morel, G., H. Panetto, M.B. Zaremba and F. Mayer (2003). Manufacturaing enterprise control and management system engineering: paradigms and open issues. IFAC Annual Review in Control 27, 199-209.

Morel, G., P. Valckenaers, J-M. Faure, C. Pereira and C. Dietrich (2005). Survey paper on manufacturing plan control challenges and issues. In: 16th IFAC World Congress. Prague, Czech Republic.

Pannequin, R. and A. Thomas (2006). Proposition of an experimental platform for product-driven systems. In: $6^{\circ}$ Conference Francophone de MOdélisation et SIMulation. Rabat, Morocco (in french).

Simon, H.A. (1996). The sciences of the artificial. third ed.. The MIT Press.

Valckenaers, P., H. van Brussel, Hadeli, O. Bochmann, B. Saint Germain and C. Zamfirescu (2003). On the design of emergent systems: an investigation of integration and interoperability issues. Engineering Applications of Artificial Intelligence 16, 377-393.

van Brussel, H., J. Wyns, P. Valckenaers, L. Bongaerts and P. Peeters (1998). Reference architecture for holonic manufacturing systems: Prosa. Computer in Industry 37, 255-274.

Vollman, T.E., W.L. Berry and D.C. Whybark (1997). Manufacturing Planning and Control Systems. fourth ed.. Irwin. 